\title{
Fully Printed Organic-Inorganic Nanocomposites for Flexible Thermoelectric Applications
}

\author{
Canlin Ou, ${ }^{\dagger}$ Abhijeet L. Sangle, ${ }^{\dagger}$ Anuja Datta, ${ }^{\dagger}$ Qingshen Jing, ${ }^{\dagger}$ Tommaso Busolo, $^{\dagger}$ Thomas Chalklen, $^{\dagger}$
} Vijay Narayan, ${ }^{\ddagger}$ and Sohini Kar-Narayan* ${ }^{\dagger}$ (1)

${ }^{\dagger}$ Department of Materials Science \& Metallurgy, University of Cambridge, 27 Charles Babbage Road, Cambridge CB3 0FS, U.K.

${ }^{\ddagger}$ Department of Physics, Cavendish Laboratories, University of Cambridge, J. J. Thompson Avenue, Cambridge, CB3 0HE, U.K.

\section{Supporting Information}

ABSTRACT: Thermoelectric materials, capable of interconverting heat and electricity, are attractive for applications in thermal energy harvesting as a means to power wireless sensors, wearable devices, and portable electronics. However, traditional inorganic thermoelectric materials pose significant challenges due to high cost, toxicity, scarcity, and brittleness, particularly when it comes to applications requiring flexibility. Here, we investigate organic-inorganic nanocomposites that have been developed from bespoke inks which are printed via an aerosol jet printing method onto flexible substrates. For this purpose, a novel in situ aerosol mixing method has been developed to ensure uniform distribution of $\mathrm{Bi}_{2} \mathrm{Te}_{3}$ / $\mathrm{Sb}_{2} \mathrm{Te}_{3}$ nanocrystals, fabricated by a scalable solvothermal synthesis method, within a poly(3,4-ethylenedioxythiophene) polystyrene sulfonate matrix. The thermoelectric properties of the resulting printed nanocomposite structures have been evaluated as a function of composition, and the power factor was found to be maximum $\left(\sim 30 \mu \mathrm{W} / \mathrm{mK}^{2}\right)$ for a nominal loading fraction of 85 wt $\%$ $\mathrm{Sb}_{2} \mathrm{Te}_{3}$ nanoflakes. Importantly, the printed nanocomposites were found to be stable and robust upon repeated flexing to curvatures up to $300 \mathrm{~m}^{-1}$, making these hybrid materials particularly suitable for flexible thermoelectric applications.

KEYWORDS: thermoelectric nanocomposite, poly(3,4-ethylenedioxythiophene) polystyrene sulfonate, solvothermal synthesis, aerosol jet printing, energy harvesting

\section{INTRODUCTION}

Harvesting energy from light, vibrations, thermal and biological sources in our environment has drawn enormous attention owing to its potential of offering a fundamental energy solution for "small power" applications such as wireless electronics, wearable devices and biomedical implants. ${ }^{1,2}$ Thermal energy harvesting has drawn considerable interest as there are several sources of heat in our environment, a major part of which is wasted, such as the heat from exhausts and radiators, industrial processes, or even that arising from the temperature difference between the human body and the ambient environment. ${ }^{3}$ Thermoelectric generators (TEGs) can convert waste heat from the ambient environment into usable electricity, based on the principle of the Seebeck effect, which in turn can help to improve energy efficiency and reduce $\mathrm{CO}_{2}$ emissions of fossil fuel systems through waste heat recovery. In particular, there has been tremendous interest in the development of low-cost, flexible, and efficient thermoelectric devices for thermal energy harvesting to power wireless sensors, wearable technology gadgets, and extreme weather clothing from human body heat. $^{4-8}$

The development of new thermoelectric materials remains a key challenge, particularly in light of the scarcity and toxicity of

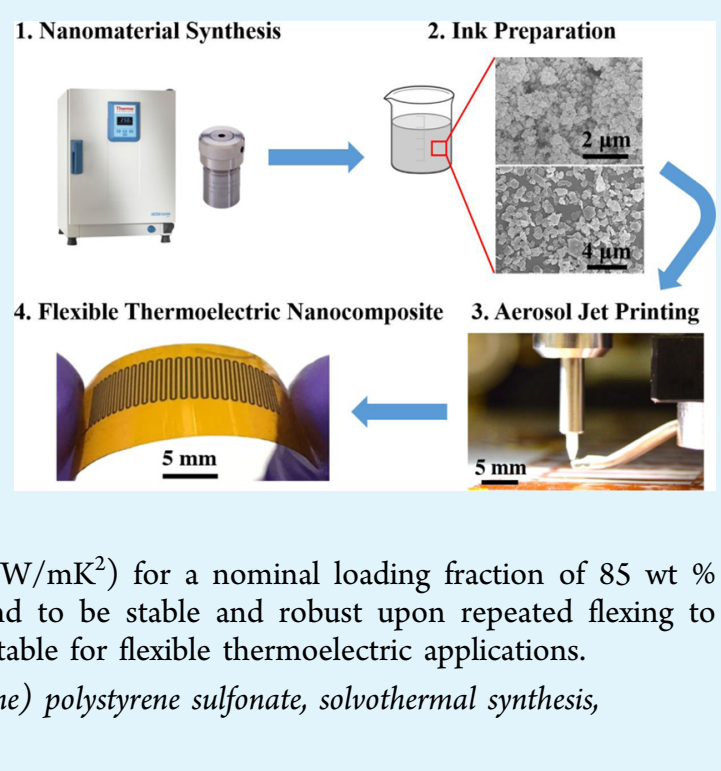

conventional inorganic thermoelectric materials. As of now, most well-established thermoelectric materials are typically metallic alloys, semiconductors, or metal oxides that are heavily doped. $^{9-12}$ Among them, binary bulk chalcogenides such as bismuth telluride $\left(\mathrm{Bi}_{2} \mathrm{Te}_{3}\right)$, antimony telluride $\left(\mathrm{Sb}_{2} \mathrm{Te}_{3}\right)$, and their alloys have been reported as the best thermoelectric materials near room temperature, making them the preferred choice for most thermoelectric applications. ${ }^{3,9}$ However, because of the scarcity, brittleness, and poor processability of these bulk chalcogenides, ${ }^{13}$ their applications are largely limited particularly when it comes to integration into flexible and/or wearable devices. ${ }^{4,5,7}$ Organic semiconductors, on the other hand, are particularly well suited for potentially low-cost and flexible thermoelectric applications, although their thermoelectric performance still lags behind their inorganic counterparts. ${ }^{14-16}$ Among the most recently reported thermoelectric polymers, p-type poly(3,4-ethylenedioxythiophene) polystyrene sulfonate (PEDOT:PSS) was shown to exhibit the best thermoelectric properties as reported by Kim et al. ${ }^{17}$

Received: January 25, 2018

Accepted: May 18, 2018

Published: May 18, 2018 
PEDOT:PSS is also one of the most well-researched and commercially used conducting polymers because of its good electrical conductivity $(\sigma)$ and environmental stability. ${ }^{18,19}$ Although its Seebeck coefficient $(S)$ is relatively low compared with inorganic counterparts, and its applications are limited to a lower temperature $(T)$ range, ${ }^{13}$ it is relatively low-cost, lightweight, non-toxic, abundant, and easily processable with inherently low thermal conductivity $(\kappa),{ }^{20}$ which is desirable to achieve a good thermoelectric figure-of-merit $Z T$ defined as $S^{2} \sigma T / \kappa^{21}$ Importantly, PEDOT:PSS is suitable for large-area deposition or printing techniques that are associated with highvolume manufacturing of printed organic electronics. ${ }^{22-25}$

In this work, we investigate the effect of inclusion of nanoparticulate $\mathrm{Bi}_{2} \mathrm{Te}_{3}$ and $\mathrm{Sb}_{2} \mathrm{Te}_{3}$ on the thermoelectric properties of aerosol jet printed PEDOT:PSS-based inks. We show that this approach can be an effective way of enhancing both $S$ and $\sigma$ (and hence the power factor, $\mathrm{PF}=S^{2} \sigma$ ) in the organic-inorganic hybrid material thus formed. Furthermore, it has already been shown by both experimental and theoretical results that nanostructuring can be an effective approach to achieve much higher $Z T$ values than can be achieved in the bulk, ${ }^{4,26-29}$ by splitting the interdependence of the electrical and thermal transport, ${ }^{28}$ thereby tuning the contribution of thermal carriers (phonons) and charge carriers (electrons) in the system. Therefore, a novel hybrid organic-inorganic nanocomposite structure is beneficial because of the intrinsically low $\kappa$ polymeric matrix, ${ }^{19,30}$ as well as the introduced phonon-boundary scattering and the large thermal boundary resistance at the interfaces that can synergistically hinder thermal transport, thereby significantly lowering the $\kappa$ of the nanocomposite. ${ }^{23,30,31}$ In this work, inorganic nanoparticulate inks have been specifically developed through solvothermal synthesis of high-quality $\mathrm{Bi}_{2} \mathrm{Te}_{3}$ and $\mathrm{Sb}_{2} \mathrm{Te}_{3}$ nanocrystals, which were then combined with commercially available PEDOT:PSS to produce printed nanocomposite structures on flexible substrates.

Organic-inorganic nanocomposites have been previously studied; however, these have been predominantly in the form of thin films grown on rigid substrates, ${ }^{6,27,32-36}$ which are inappropriate for flexible, conformable, and/or wearable electronic applications. In this regard, large-area printing technologies, such as inkjet printing, ${ }^{18,37,38}$ screen printing, ${ }^{39-41}$ dispenser printing or stereolithography, ${ }^{42-44}$ offer the scope for miniaturization of flexible thermoelectric nanocomposite devices for small-scale energy harvesting technologies. ${ }^{45-47}$ However, in this work, we used the aerosol jet printing (AJP) technique to print bespoke organicinorganic nanocomposite inks as this technique possesses several advantages over other printing techniques. First, it is a promising scalable direct-write deposition technique for developing next generation printed flexible and/or stretchable micrometer-scale electronics. This is because it enables largearea deposition of fine-scale features from a wide range of functional inks onto various planar or nonplanar substrates. AJP supports a wide variety of commercially available electronic materials and custom formulations, such as conductive inks, polymers, and so forth. ${ }^{48}$ Secondly, it involves a high-precision controlled deposition process with minimal loss of material, which is advantageous when dealing with small quantities of expensive materials. Finally, functional inks over a wide range of viscosities can be printed layer-by-layer onto both rigid and flexible substrates. ${ }^{48}$
The AJP technique involves creating an "aerosol" of ink and using a carrier gas to propel this aerosol toward a substrate. The printer used in this work (Optomec AJP 200) supports two atomization methods via an ultrasonic atomizer (UA) and a pneumatic atomizer (PA) (see Supporting Information S1), which are used to atomize the liquid-source ink into a dense aerosol mist composed of highly loaded droplets ranging from 1 to $5 \mu \mathrm{m}$ in diameter. These droplets are transported within a $\mathrm{N}_{2}$ carrier gas and directly fed toward the deposition print head via aerodynamic focusing involving a sheath gas for printing features with controlled precision. ${ }^{41}$ To realize printed organic-inorganic nanocomposites, we have developed a novel in situ mixing method where the organic and inorganic inks are separately atomized in the UA and PA, respectively, and the resulting aerosol mists are mixed uniformly en route to the nozzle prior to deposition onto a flexible polyimide substrate. The morphology and thermoelectric properties of the resulting printed structures have been studied as a function of the loading fraction of the inorganic component to identify the optimum composition for enhanced PF. Importantly, we directly demonstrate the suitability of the printed nanocomposite structures for applications in flexible thermoelectric energy harvesters, through a detailed study of how the relevant thermoelectric properties vary upon flexing to curvatures up to $300 \mathrm{~m}^{-1}$

\section{RESULTS AND DISCUSSION}

2.1. Solvothermal Synthesis of $\mathrm{Bi}_{2} \mathrm{Te}_{3}$ and $\mathrm{Sb}_{2} \mathrm{Te}_{3}$ Nanocrystals. Bismuth telluride $\left(\mathrm{Bi}_{2} \mathrm{Te}_{3}\right)$ and antimony telluride $\left(\mathrm{Sb}_{2} \mathrm{Te}_{3}\right)$ nanocrystals were synthesized via a facile and scalable solvothermal synthesis process. This promising low-temperature approach has been shown to achieve high yield with good control on composition, phase purity, shape, and size distribution. ${ }^{28,49}$ The ethylene glycol (EG) mediated solvothermal synthesis method adopted in this work involves a redox reaction between the bismuth nitrate, antimony acetate and sodium tellurite. ${ }^{28}$ Additionally, no complex organic ligands or templates were used in this synthesis method to confine the nanocrystals into different shapes. ${ }^{28}$ The synthesis process and the morphological and microstructural characterization are discussed comprehensively in the Experimental Section.

Scanning electron microscopy (SEM) images shown in Figure $1 \mathrm{a}, \mathrm{b}$ revealed a spherical nanoparticle form of the assynthesized $\mathrm{Bi}_{2} \mathrm{Te}_{3}$ with an average particle size of $\sim 129 \mathrm{~nm}$ and a size distribution of $\pm 49 \mathrm{~nm}$, while $\mathrm{Sb}_{2} \mathrm{Te}_{3}$ nanocrystals
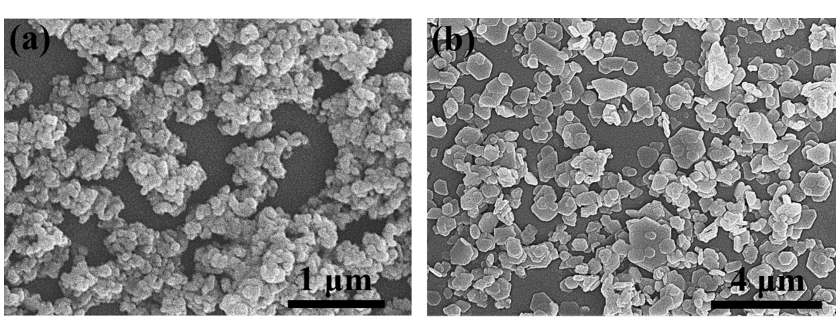

(c)

\begin{tabular}{|c|c|}
\hline Element & $\begin{array}{c}\text { Atomic } \\
\text { Concentration [at.\%] }\end{array}$ \\
\hline Bismuth (Bi) & $41.1(2.2)$ \\
\hline Tellurium (Te) & $\mathbf{5 8 . 9 ( 2 . 1 )}$ \\
\hline $\mathrm{Bi}: \mathrm{Te}$ & $\approx 2.1: 3$ \\
\hline
\end{tabular}

(d)

\begin{tabular}{|c|c|}
\hline Element & $\begin{array}{c}\text { Atomic } \\
\text { Concentration [at.\%] }\end{array}$ \\
\hline Antimony (Sb) & $39.6(1.9)$ \\
\hline Tellurium (Te) & $\mathbf{6 0 . 4 ( 2 . 9 )}$ \\
\hline $\mathrm{Sb}: \mathrm{Te}$ & $\approx 1.9: 3$ \\
\hline
\end{tabular}

Figure 1. SEM images of solvothermal-synthesized (a) $\mathrm{Bi}_{2} \mathrm{Te}_{3}$ nanoparticles and (b) $\mathrm{Sb}_{2} \mathrm{Te}_{3}$ nanoflakes. EDX compositional analysis of (c) $\mathrm{Bi}_{2} \mathrm{Te}_{3}$ nanoparticles and (d) $\mathrm{Sb}_{2} \mathrm{Te}_{3}$ nanoflakes. 
were found to be randomly oriented in thin flake form with an average lateral diameter of $\sim 534 \mathrm{~nm}$ and a size distribution of $\pm 281 \mathrm{~nm}$. The flake-like structure is commonly observed in $\mathrm{Sb}_{2} \mathrm{Te}_{3}$ because of its inherent anisotropic and layered crystal structure. $^{28}$ Energy dispersive X-ray (EDX) analysis on these synthesized nanocrystals showed the actual "Bi" and "Te" atomic concentration in the $\mathrm{Bi}_{2} \mathrm{Te}_{3}$ sample to be $41.1 \%$ and $58.9 \%$, respectively, and the actual " $\mathrm{Sb}$ " and "Te" atomic concentration in the $\mathrm{Sb}_{2} \mathrm{Te}_{3}$ sample to be $39.6 \%$ and $60.4 \%$, as tabulated in Figure 1c,d. By controlling the $\mathrm{Bi} / \mathrm{Te}$ and $\mathrm{Sb} / \mathrm{Te}$ ratio in the precursor solution, the synthesized composition can be adjusted. If $\mathrm{Bi}_{2} \mathrm{Te}_{3} / \mathrm{Sb}_{2} \mathrm{Te}_{3}$ were to be $\mathrm{Bi}$-rich/Sb-rich or Tedeficient, the $\mathrm{Bi} / \mathrm{Sb}$ would contribute to more hole carriers $\left(\mathrm{h}^{+}\right)$ and exhibit p-type properties or vice versa. ${ }^{28}$ Our synthesized $\mathrm{Bi}_{2} \mathrm{Te}_{3}$ exhibited slightly higher "Bi" at $\%$ than the stoichiometric amount, leading to Bi-rich material that is hole carrier dominated, while the $\mathrm{Sb}_{2} \mathrm{Te}_{3}$ was nearly stoichiometric.

$\mathrm{X}$-ray diffractometry (XRD) studies presented in Figure 2a,b showed that (015) was the sharpest and most intense

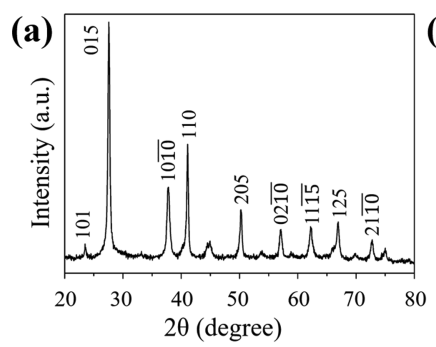

(c)

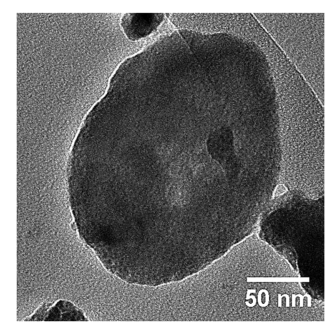

(e)
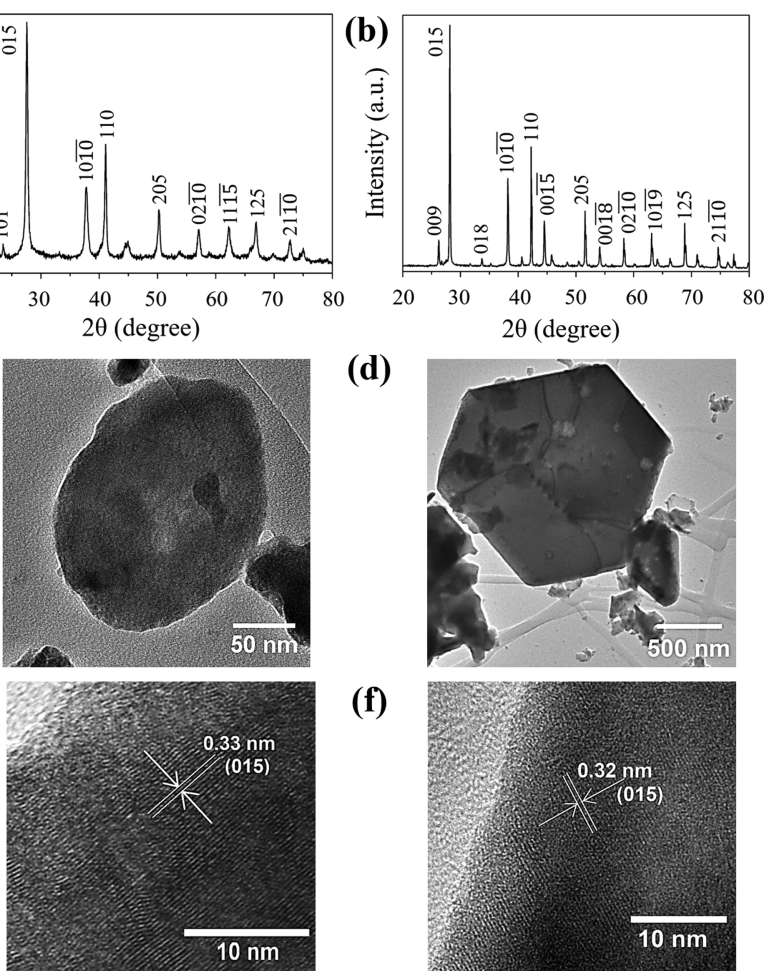

(d)

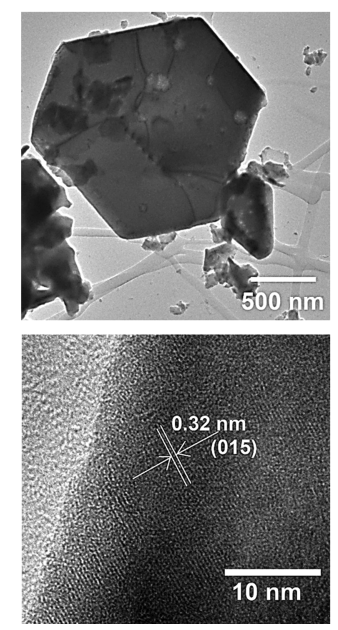

Figure 2. XRD results of solvothermal-synthesized (a) $\mathrm{Bi}_{2} \mathrm{Te}_{3}$ nanoparticles and (b) $\mathrm{Sb}_{2} \mathrm{Te}_{3}$ nanoflakes. TEM analysis of (c) $\mathrm{Bi}_{2} \mathrm{Te}_{3}$ nanoparticle and (d) $\mathrm{Sb}_{2} \mathrm{Te}_{3}$ nanoflake, and the lattice fringe patterns of (e) $\mathrm{Bi}_{2} \mathrm{Te}_{3}$ nanoparticle and (f) $\mathrm{Sb}_{2} \mathrm{Te}_{3}$ nanoflake, respectively.

diffraction plane for both $\mathrm{Bi}_{2} \mathrm{Te}_{3}$ and $\mathrm{Sb}_{2} \mathrm{Te}_{3}$ as expected, with no detectable impurity peaks, indicating that a good crystalline quality was obtained. The $\mathrm{Bi}_{2} \mathrm{Te}_{3}$ and $\mathrm{Sb}_{2} \mathrm{Te}_{3}$ XRD spectra were both indexed with rhombohedral symmetry (space group $R \overline{3} m$ ) and indicated an average crystallite size of $\sim 201$ $( \pm 53) \mathrm{nm} \sim 479( \pm 59) \mathrm{nm}$, respectively, as calculated by the Debye-Scherrer equation. ${ }^{50}$ These calculated crystallite sizes were within reasonable agreement with the average particle sizes estimated from the SEM images. The crystal structure of the as-prepared $\mathrm{Bi}_{2} \mathrm{Te}_{3}$ and $\mathrm{Sb}_{2} \mathrm{Te}_{3}$ nanocrystals were investigated by high-resolution transmission electron micros- copy (HRTEM) shown in Figure $2 \mathrm{c}-\mathrm{f}$. The polycrystalline nature of $\mathrm{Bi}_{2} \mathrm{Te}_{3}$ nanoparticles (Figure $2 \mathrm{c}$ ) was revealed by the discontinuous lattice fringes oriented in the same direction in Figure 2e. The $d$-spacing of $0.33 \mathrm{~nm}$ was in good agreement with the (015) interplaner spacing of $\mathrm{Bi}_{2} \mathrm{Te}_{3}$. Hexagonal-shaped $\mathrm{Sb}_{2} \mathrm{Te}_{3}$ nanocrystals (Figure $2 \mathrm{~d}$ ) showed more continuous lattice fringes as compared to $\mathrm{Bi}_{2} \mathrm{Te}_{3}$ nanoparticles. The lattice fringe spacing of $0.32 \mathrm{~nm}$ was also in agreement with (015) interplanar spacing in hexagonal $\mathrm{Sb}_{2} \mathrm{Te}_{3}$ (see Figure $2 \mathrm{f}$ ).

2.2. Characterization and Measurement of Printed PEDOT:PSS-Based Nanocomposites. Organic-inorganic nanocomposites comprising commercially available $\mathrm{PE}$ DOT:PSS and solvothermal-synthesized $\mathrm{Bi}_{2} \mathrm{Te}_{3} / \mathrm{Sb}_{2} \mathrm{Te}_{3}$ nanocrystals were directly printed onto a flexible polyimide sheet via the AJP method. The detailed fabrication method is discussed in the Experimental Section and Supporting Information S1, where a novel in situ mixing method was applied for better control of the mixing between the organic polymer ink and inorganic nanoparticulate ink, as illustrated schematically in Figure 3a. The flexibility and robustness of the printed

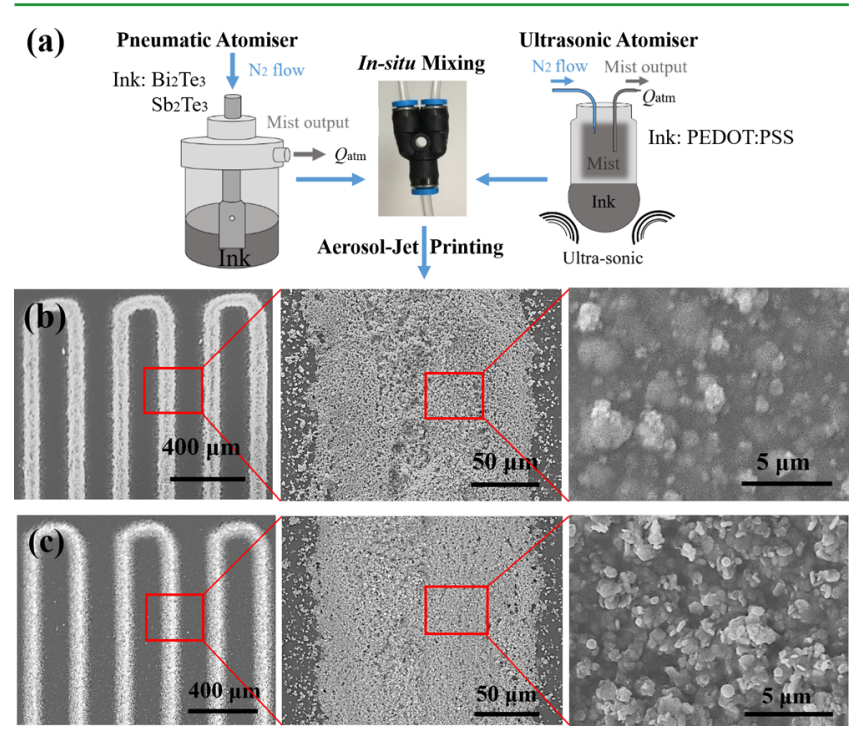

Figure 3. (a) Schematic of the novel in situ mixing method for nanocomposite printing by AJP. SEM images of five-layer printed PEDOT:PSS-based nanocomposites loaded with (b) $50 \mathrm{wt} \%$ (nominal) $\mathrm{Bi}_{2} \mathrm{Te}_{3}$ nanoparticles and (c) 85 wt \% (nominal) $\mathrm{Sb}_{2} \mathrm{Te}_{3}$ nanoflakes, where the enlarged images show details of their morphology and microstructure.

nanocomposite were realized by using a novel oscillatory geometrical architecture as shown in Figure S1, while the polyimide substrate provided both flexibility and stability at temperatures up to $400{ }^{\circ} \mathrm{C}$.

To start with, preliminary experiments were conducted to investigate the influence of different surface treatments, namely $\mathrm{H}_{2} \mathrm{SO}_{4}$, EG, and dimethyl sulfoxide, on the as-printed pristine PEDOT:PSS structures as discussed in Supporting Information S2. In the literature, various surface treatments have been conducted to de-dope the insulating PSS molecules from the conducting PEDOT chains, to form better electrical contacts between neighboring PEDOT chains. ${ }^{17,51-53}$ Separately, it was found that denser and more uniform nanocomposite structures with fewer noticeable pores or cracks on the surface were obtained with increasing number of printed layers, leading to homogeneous printed structures of thicknesses ranging 
between 1 and $2 \mu \mathrm{m}$ and widths ranging between 150 and 180 $\mu \mathrm{m}$, depending on the composition (Figures $3 \mathrm{~b}, \mathrm{c}$ and S6). In our case, it was found that EG-treated samples with five-layer printing exhibited the highest $\sigma$ and PF (see Figure S3). Hence, EG was chosen for all subsequent surface treatment of printed structures that were realized through five-layer printing of the nanocomposites, where $\mathrm{Bi}_{2} \mathrm{Te}_{3} / \mathrm{Sb}_{2} \mathrm{Te}_{3}$ nanocrystals were introduced in a controlled fashion by varying the loading weight fraction during the in situ mixing stage of the AJP process. The printing quality and morphology of the PEDOT:PSS-based nanocomposites are discussed in more detail in Supporting Information S3 and S4.

Although the PEDOT:PSS polymer has relatively lower $S$ compared with their inorganic counterparts, the overall PF of a composite comprising the two can be modified by varying the loading ratio between the organic and the inorganic components to overcome the limitations of the constituent materials. ${ }^{6,23,54}$ With the aid of the in situ mixing method (Figures $3 \mathrm{a}$ and S2), different loading weight percentage (wt \%) of solvothermal-synthesized $\mathrm{Bi}_{2} \mathrm{Te}_{3}$ nanoparticles and $\mathrm{Sb}_{2} \mathrm{Te}_{3}$ nanoflakes, from 0 to 100 wt \% (nominal), were, respectively, blended within the PEDOT:PSS matrix to investigate the effect of nanoparticle loading ratio on the thermoelectric properties of printed PEDOT:PSS-based nanocomposites. The morphology of two representative printed nanocomposite structures are shown in the SEM images in Figure $3 b, c$, showing uniformly distributed and embedded $\mathrm{Bi}_{2} \mathrm{Te}_{3}$ and $\mathrm{Sb}_{2} \mathrm{Te}_{3}$ nanocrystals, respectively, within the PEDOT:PSS polymer matrix. The nanocomposites exhibited a close-packed microstructure and a 3D conducting network with little clumping and improved mechanical strength, which was consistent across all different compositions studied (Figure S6).

Figure $4 a-c$ compares the thermoelectric properties of different printed PEDOT:PSS-based nanocomposites (both treated with EG and nontreated) as measured using a bespoke thermoelectric experimental setup described in the Experimental Section and Supporting Information S5. It can be seen that in all cases, surface treatment with EG on PEDOT:PSSbased nanocomposites improved $\sigma$ in particular and was thus essential to enhance the thermoelectric properties. It was found that overall, $S$ values increased with increasing loading ratio of $\mathrm{Bi}_{2} \mathrm{Te}_{3}$ and $\mathrm{Sb}_{2} \mathrm{Te}_{3}$, while $\sigma$ slightly decreased on average, which might be attributed to the contact resistance from the interfaces between PEDOT:PSS and $\mathrm{Bi}_{2} \mathrm{Te}_{3}$ or $\mathrm{Sb}_{2} \mathrm{Te}_{3}$ nanocrystals. If both components are either $\mathrm{n}$ - or p-type, they work synergistically to enhance the $\sigma$. However, if both components present opposing carrier types, the final thermoelectric performance of the nanocomposite would depend on the relative ratio of the constituents. ${ }^{6,23}$ The $S$ of $\mathrm{Bi}_{2} \mathrm{Te}_{3}$-loaded nanocomposite largely increased with the increasing loading ratio and peaked at $\sim 36.6 \mu \mathrm{V} / \mathrm{K}$ compared with the pristine PEDOT:PSS film with $S$ of $\sim 17.1 \mu \mathrm{V} / \mathrm{K}$, which confirmed that the solvothermal-synthesized $\mathrm{Bi}_{2} \mathrm{Te}_{3}$ nanoparticles exhibited ptype behavior that matched with the EDX result in Figure 1c. It should be mentioned that the $S$ of the printed pristine PEDOT:PSS film here was relatively lower than the reported values in the literature, ${ }^{17}$ which might be due to the use of a different commercial supplier, as discussed in Supporting Information S2. From our measurements, the fact that $\sigma$ of these nanocomposites slightly decreased with increasing loading wt \% while $S$ was largely enhanced and remained at relatively high level suggests that $S$ and $\sigma$ could be decoupled to maximize the final PF. The actual variation of $\sigma$ with
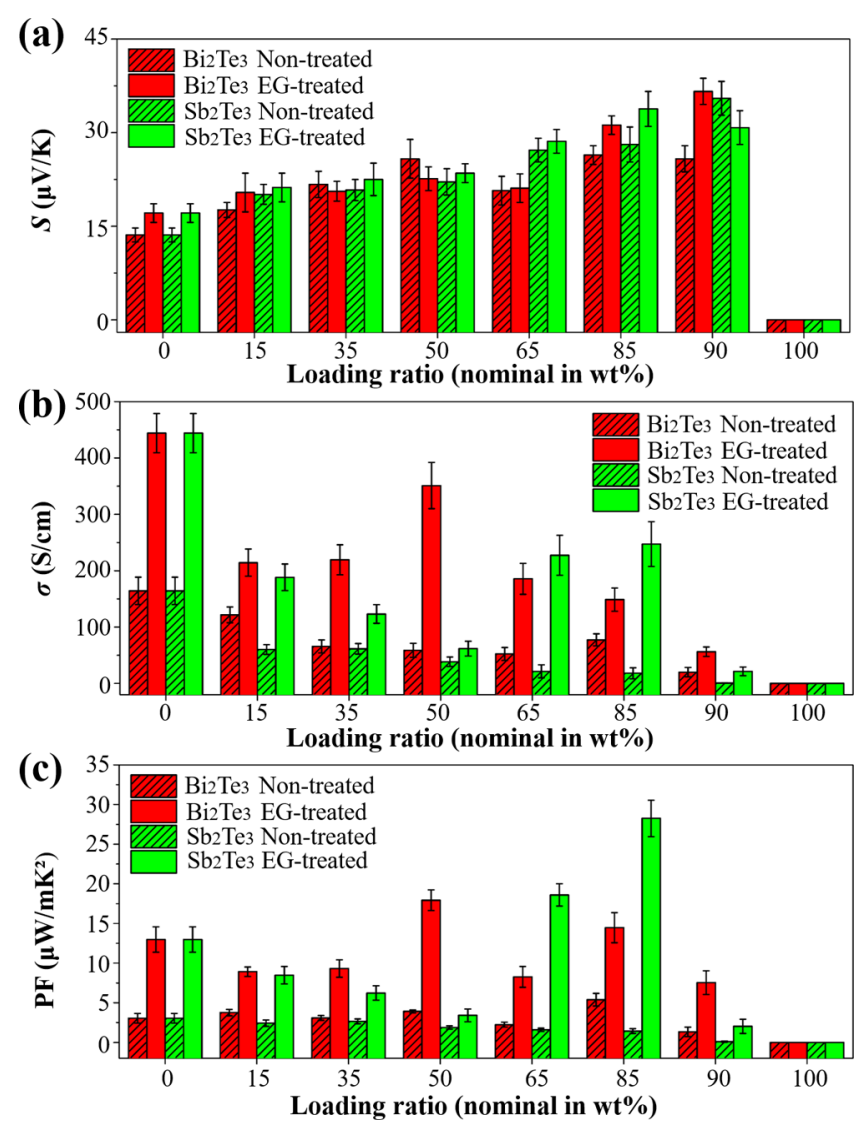

Figure 4. (a) Seebeck coefficient, (b) electrical conductivity, and (c) PF of nontreated and EG-treated five-layer printed PEDOT:PSS-based nanocomposites loaded with different wt $\%$ of $\mathrm{Bi}_{2} \mathrm{Te}_{3}$ nanoparticles and $\mathrm{Sb}_{2} \mathrm{Te}_{3}$ nanoflakes, respectively. Error bars indicate the standard deviation of the measured values of two separate printed structures with the same loading ratio.

composition is in reality more complex. For low loading wt $\%$, the introduction of organic-inorganic interfaces led to a decrease in $\sigma$, however, with increasing loading wt $\%$, the aggregation of the inorganic nanocrystals into a continuous $3 \mathrm{D}$ conducting network initially led to a slight increase in $\sigma$, but continued aggregation led to a higher proportion of loaded particles not electrically connected by the PEDOT:PSS matrix, thus leading to a drop in $\sigma$ at the highest loading wt \%. The trend of this variation is slightly different for $\mathrm{Bi}_{2} \mathrm{Te}_{3}$-based and $\mathrm{Sb}_{2} \mathrm{Te}_{3}$-based nanocomposites because of the inherently different nanocrystal sizes and morphologies (nanoparticles vs nanoflakes) giving rise to different aggregation characteristics.

In general, it was found that our printed nanocomposites were stable under ambient conditions over several weeks of testing. The best thermoelectric performance of the printed ptype PEDOT:PSS-based nanocomposites was achieved at $85 \mathrm{wt}$ $\% \mathrm{Sb}_{2} \mathrm{Te}_{3}$, with $S$ of $\sim 33.8 \mu \mathrm{V} / \mathrm{K}, \sigma$ of $\sim 247.3 \mathrm{~S} / \mathrm{cm}$, and $\mathrm{PF}$ of $\sim 28.3 \mu \mathrm{W} / \mathrm{mK}^{2}$, which is comparable to what has been reported in the literature. ${ }^{22,23,55-57}$ The $100 \mathrm{wt} \%$ samples refer to aqueous dispersions of $\mathrm{Bi}_{2} \mathrm{Te}_{3}$ or $\mathrm{Sb}_{2} \mathrm{Te}_{3}$ printed onto the substrate without the presence of the PEDOT:PSS matrix. The thermoelectric properties of these samples were found to be far inferior to the printed nanocomposites because of the relatively poor connectivity between the nanoparticles in the absence of PEDOT:PSS. The connectivity could have been improved by sintering at elevated temperature, but this was avoided because of the presence of the plastic substrate. Additionally, we 
performed finite element modeling (COMSOL Multiphysics) to simulate the effect of an imposed temperature gradient across the printed thermoelectric nanocomposite which yielded a good match with the experimental measurement discussed in the Supporting Information S6, as shown in Figure S8.

2.3. Flexibility Measurement of Printed PEDOT:PSSBased Nanocomposites. Thus far, considerable research has been conducted toward the enhancement of PF or ZT values in existing classes of thermoelectric materials, ${ }^{17,21,31,58,59}$ but the mechanical properties and flexibility of these materials when incorporated into thermoelectric devices modules are severely under-represented in the literature. To address this issue, we devised a simple manual bending test on our printed PEDOT:PSS-based nanocomposite structures to assess their thermoelectric properties under various degrees of curvature, as shown in Figure $5 a-c$. An excellent conformability of the
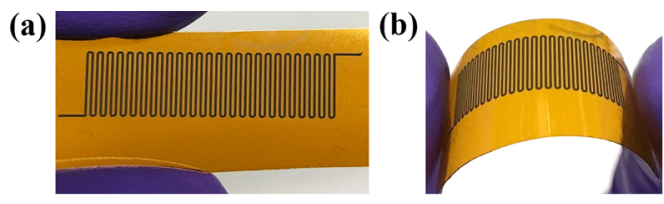

(c)

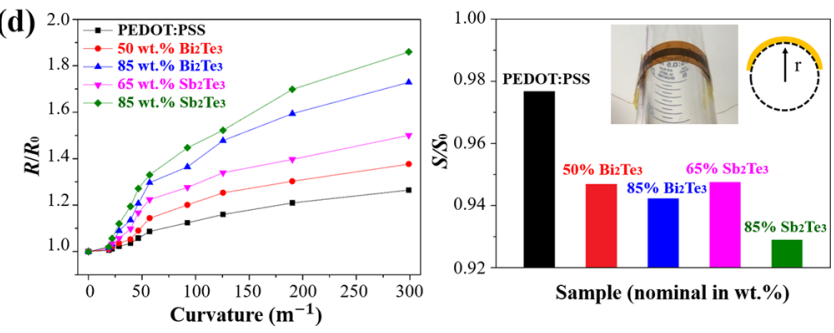

Figure 5. Photograph of the thermoelectric nanocomposite printed onto a flexible polyimide sheet, bent to different degrees: (a) flat, (b) lower degree, and (c) higher degree. (d) Flexing test on the printed PEDOT:PSS-based nanocomposites with different loading ratios of $\mathrm{Bi}_{2} \mathrm{Te}_{3}$ and $\mathrm{Sb}_{2} \mathrm{Te}_{3}$, where the ratio of flat-to-flexed resistance was plotted as a function of curvature and the ratio of flat-to-flexed Seebeck coefficient as a function of the loading ratio, respectively. Inset: the sample being subjected to a curvature of $190 \mathrm{~m}^{-1}$.

printed nanocomposite was observed, and they retained their original shape and smooth surface without forming any visible cracks or deformations after several hand-bending tests, and their electrical properties remained nearly the same throughout. Further rigorous flexing tests were subsequently conducted on these printed nanocomposites to estimate their operation range. The samples were bent by being mounted on different surfaces having different curvatures, such as a beaker, test tube, pen, and so forth, and room temperature electrical resistance was measured simultaneously. The ratio of flat-to-flexed resistance was plotted as a function of curvature, and the nanocomposites with relatively higher PF values were selectively compared. The effect of flexing on $S$ was also determined by measuring this before and after flexing, as shown in Figure 5d. Electrical resistance was selected here as the criterion to evaluate the film flexibility, as this is expectedly sensitive to any cracks or voids that may be introduced during the flexing test. ${ }^{48}$ As the curvature increased, the electrical resistance of the nanocomposites slightly increased and then remained relatively stable under very high curvature, indicating their superior flexibility. However, for all samples that were flextested, their electrical resistance values did not come back to their original points after release, which may be due to the formation of disconnections (possibly in the form of cracks) within the nanocomposite. The $S / S_{0}$ ratio only decreased slightly after being flexed to very high curvatures (up to 300 $\mathrm{m}^{-1}$ ), which also confirmed the excellent flexibility and mechanical strength of these printed nanocomposites. The overall PF of the best-performing nanocomposite structure was found to reduce by half, which meant the device was still useable as a TEG. These flexing results suggest that our novel nanocomposite design structure, comprising fine-grained nanocrystals within a ductile thermoelectric polymer serving as a protective matrix, can offer greater mechanical support and protection, whilst maintaining relatively good electrical conductivity between the nanocrystals. These printed nanocomposites are therefore more flexible and robust than their bulk counterparts which are normally produced by the directional solidification process. ${ }^{29,60}$ Notably, our in situ mixing method required only small amounts of organic and inorganic constituent materials because of the nature of AJP being an additive-manufacturing technique. The final nanocomposites could be directly incorporated into thermoelectric applications with minimal post-processing treatment. We demonstrated this by designing a simple printed and flexible TEG, as discussed in Supporting Information S7 and shown in Figure S9, that could be used as a flexible "thermoelectric coaster" to convert the temperature difference between a can of hot/cold liquid and the ambient environment into electricity.

\section{CONCLUSION}

In summary, a versatile, cost-effective and easily scalable AJP technique for depositing p-type PEDOT:PSS-based nanocomposites onto flexible substrates has been successfully developed with the combination of our in-house simple, highyield and highly scalable solvothermal synthesis method for $\mathrm{Bi}_{2} \mathrm{Te}_{3}$ nanoparticle and $\mathrm{Sb}_{2} \mathrm{Te}_{3}$ nanoflake synthesis. This lowtemperature solution-based synthesis approach allowed good control of size and shape at the nanoscale, and thus enabled better control and enhancement of thermoelectric properties of the resulting PEDOT:PSS-based nanocomposites. Different weight percentages of solvothermal-synthesized nanostructured $\mathrm{Bi}_{2} \mathrm{Te}_{3}$ and $\mathrm{Sb}_{2} \mathrm{Te}_{3}$ particles with high $S$ and high $\sigma$ were integrated with the low $\kappa$ conducting polymers PEDOT:PSS, for printing thermoelectric nanocomposites onto a cheap and flexible polyimide sheet via AJP method. The PEDOT:PSSbased nanocomposites loaded nominally with $85 \mathrm{wt} \% \mathrm{Sb}_{2} \mathrm{Te}_{3}$ nanoflakes exhibited the best thermoelectric performance $(S \approx$ $33.8 \mu \mathrm{V} / \mathrm{K}, \sigma \approx 247.3 \mathrm{~S} / \mathrm{cm}$ and $\mathrm{PF} \approx 28.3 \mu \mathrm{W} / \mathrm{mK}^{2}$ ) as well as excellent mechanical properties. The flexibility of the asprinted nanocomposite structures was rigorously tested and showed stable and robust performance upon repeated flexing. The demonstration of flexibility enables broader applications of these printed organic-inorganic nanocomposites, particularly for wearable electronic device applications.

\section{EXPERIMENTAL SECTION}

4.1. Synthesis of Thermoelectric Nanomaterials. Bismuth nitrate pentahydrate $\left(0.25 \mathrm{mmol}, \mathrm{Bi}\left(\mathrm{NO}_{3}\right)_{3} \cdot 5 \mathrm{H}_{2} \mathrm{O}, 98.0 \%\right.$, SigmaAldrich) was first dissolved in $1 \mathrm{~mL}$ of acetic acid (99.7\%, SigmaAldrich). Then, $9 \mathrm{~mL}$ of EG (99.8\%, Sigma-Aldrich) was added as a reducing agent and capping agent to form a protective layer around the particle surfaces and inhibit the particle growth and agglomeration. $^{28,49}$ After stirring for $10 \mathrm{~min}, 0.375 \mathrm{mmol}$ of sodium tellurite $\left(\mathrm{Na}_{2} \mathrm{TeO}_{3}, 99 \%\right.$, Sigma-Aldrich) was added as the "Te" source, and stirred for $30 \mathrm{~min}$ for homogeneity. Finally, when all added powders 
were completely dissolved, $5 \mathrm{~mL}$ of hydrazine hydrate $\left(\mathrm{N}_{2} \mathrm{H}_{4} \cdot \mathrm{H}_{2} \mathrm{O}\right.$, $50-60 \%$, Sigma-Aldrich) was added as a reducing reagent to reduce the sodium tellurite and also prevent the surface oxidation of synthesized nanocrystals. $^{28}$ (Note that hydrazine is highly corrosive and toxic, and easily oxidized. Adequate safety precautions were taken during its handling.) The final mixed solution was sealed within the autoclave (Shanghai Kankun Instrument, $25 \mathrm{~mL}$ ), and the reaction was then performed at $175^{\circ} \mathrm{C}$ for $12 \mathrm{~h}$ in an oven (Thermo Scientific). For the $\mathrm{Sb}_{2} \mathrm{Te}_{3}$ synthesis, the same procedure was followed except for the antimony acetate $\left(\mathrm{C}_{6} \mathrm{H}_{9} \mathrm{O}_{6} \mathrm{Sb}, 99.99 \%\right.$, Sigma-Aldrich $)$ was used as the "Sb" source, and all reactions were carried out under the same conditions. It should be noted that all chemical reagents used here were of analytical grade and used as received without further purification. After synthesis, the obtained nanocrystals were subsequently rinsed by DI water and ethanol, and then collected by centrifugation. Between each washing, they were treated within an ultrasonic bath to thoroughly clean the nanocrystals. By doing these, the unreacted chemicals and the solvent that remained on the surface were cleaned and removed. Finally, the nanocrystals were dried in a vacuum desiccator for overnight, which provided a nonoxidizing atmosphere to prevent the oxidation of metallic alloys.

4.2. Fabrication of Flexible PEDOT:PSS-Based Thermoelectric Nanocomposites. An Optomec Aerosol Jet Printer 200 System equipped with a high-resolution camera has been used in our laboratory. ${ }^{48}$ For the printable ink of UA, the commercially available PEDOT:PSS suspension ( $1 \mathrm{wt} \%$, Heraeus Clevios PH1000) was used with the addition of 5 wt \% EG was also added to improve $\sigma$ of PEDOT:PSS. ${ }^{17,61,62}$ For the ink of PA, the solvothermal-synthesized $\mathrm{Bi}_{2} \mathrm{Te}_{3}$ and $\mathrm{Sb}_{2} \mathrm{Te}_{3}$ nanocrystals were blended within water with the weight percentage of 1 and $10 \mathrm{wt} \%$, respectively, and then constantly stirred during printing to ensure the suspension form. During the AJP process, different values of atomization flow rate were applied to obtain different loading ratios of $\mathrm{Bi}_{2} \mathrm{Te}_{3}$ or $\mathrm{Sb}_{2} \mathrm{Te}_{3}$ nanocrystals. Because of the low melting point of PEDOT:PSS polymer and the low oxidation temperature of chalcogenides metallic alloys, the printed thermoelectric nanocomposites were oven-cured at $130{ }^{\circ} \mathrm{C}$ for $30 \mathrm{~min}$ to remove water and other organic solvents. Finally, the printed PEDOT:PSS-based samples were surface-treated by dipping within a de-doping agent EG for $2 \mathrm{~h}$ submersion at $80{ }^{\circ} \mathrm{C}$ to improve their final $S$ and $\sigma$. The treated samples were rinsed thoroughly by dipping within DI water for a few minutes to remove the residual EG solvent as well as the de-doped PSS particles remaining on the surface, followed by gentle blow-drying with dry nitrogen gas. ${ }^{17,57}$

4.3. Characterization of Thermoelectric Nanomaterials and Nanocomposites. To visualize the morphology and microstructure of synthesized thermoelectric particles and to measure the dimensions of printed thermoelectric nanocomposites, the obtained particles and nanocomposites were coated with platinum by sputter-coating (K575 Sputter Coater) to form a thin electrically conducting film of platinum to minimize the charging effect during SEM imaging. Field-emission SEM (FEI Nova NanoSEM 450), operating at an accelerating voltage of $10 \mathrm{kV}$ and equipped with an energy dispersive $\mathrm{X}$-ray spectroscopy (EDX, Bruker XFlash 6100), was performed on the nanocrystals and nanocomposite samples to investigate their dimensional, morphological, and compositional properties. The EDX analysis was also conducted on the synthesized thermoelectric particles to investigate their stoichiometry and homogeneity of chemical composition at an accelerating voltage of $15 \mathrm{kV}$ and spot size of 4 . The crystallinity, crystallite size, orientation, and phase purity of synthesized thermoelectric particles were investigated using XRD studies. The powder samples were attached flat onto a zero-diffraction silicon sample holder, and then the XRD analysis was performed on a powder X-ray diffractometer (Bruker D8 ADVANCE) with a $\mathrm{Cu} \mathrm{K} \alpha \mathrm{X}$-ray tube $(\lambda=$ $1.5406 \AA)$, diffraction angle $\left(\theta, 20^{\circ}<2 \theta<80^{\circ}\right)$, and a step size of $0.05^{\circ}$ at the room temperature. The obtained intensity- $2 \theta$ spectra were analyzed with the aid of HighScore Plus software (version 4.1), and the average crystallite size was then calculated by the Debye-Scherrer Equation. $^{50}$ The crystallinity of nanoparticles and nanoflakes was confirmed using HRTEM (FEI Tecnai F20). HRTEM samples were prepared by first dispersing the synthesized powders into ethanol by ultrasonic treatment for $1 \mathrm{~min}$ to obtain a uniform dispersion, and then drop-casting 2-5 drops of the suspension onto the 300-mesh carbon-coated copper TEM grids. The TEM grids were air-dried under ambient conditions. The HRTEM images were obtained at an accelerating voltage of $200 \mathrm{kV}$. The line width of printed lines was quickly measured via a table-top SEM (Hitachi TM3000), while the thickness and line profile were measured by a stylus profilometer (Veeco DEKTAK). To obtain more precise results, at least three repeated measurements were conducted on each sample to take the average.

4.4. Thermoelectric Measurement of Printed Thermoelectric Nanocomposites. An in-house measurement setup, discussed in detail in Supporting Information S5 and Figure S7a-c, was developed for the simultaneous measurement of the in-plane Seebeck coefficient and electrical conductivity by a dynamic method. Briefly, silver electrodes were hand-deposited onto both edges of the printed thermoelectric patterns so as to have a better electrical and thermal contact with the Seebeck probes (copper wires) and thermometers (Pt-100 resistance thermometers). The samples were then placed between two temperature-controlled Peltier modules to create a temperature difference in between $\left(-60\right.$ to $\left.+60{ }^{\circ} \mathrm{C}\right)$. At the same time, the sample resistance and the generated Seebeck voltage were continuously recorded by our custom-designed semiautomatic data acquisition system with the combination of four-point probe measurement method to reduce the measurement error. Finally, the Seebeck coefficient was calculated by analyzing the linear regression slope of the $\Delta V-\Delta T$ curve as demonstrated in Figure S7d.

\section{ASSOCIATED CONTENT}

\section{Supporting Information}

The Supporting Information is available free of charge on the ACS Publications website at DOI: 10.1021/acsami.8b01456.

Detailed description of the fabrication of flexible PEDOT:PSS-based thermoelectric nanocomposites; effect of surface treatment on the thermoelectric properties of printed PEDOT:PSS films; printed PEDOT:PSSbased nanocomposite quality comparison; structure and morphology of printed PEDOT:PSS-based nanocomposites; measurement of thermoelectric properties of the flexible nanocomposites; finite element modeling of thermoelectric nanocomposites; and fabrication and testing of flexible TEGs (PDF)

\section{AUTHOR INFORMATION}

\section{Corresponding Author}

*E-mail: sk568@cam.ac.uk.

ORCID $\odot$

Abhijeet L. Sangle: 0000-0003-0848-4583

Sohini Kar-Narayan: 0000-0002-8151-1616

\section{Author Contributions}

C.O. fabricated the samples and performed most of the measurements reported, with input from T.B. and T.C. S.K.-N. designed and guided the experimental work, and V.N. provided guidance for the device design and measurement setups. A.D. conducted the high-resolution TEM imaging. A.L.S. built the thermoelectric measurement set-up with the help and guidance of V.N. and S.K.-N. Q.J. provided guidance on the thermal modeling section. C.O. and S.K.-N. co-wrote the paper. All authors discussed the results and commented on the paper.

\section{Notes}

The authors declare no competing financial interest.

Supporting data for this paper is available at the DSpace@ Cambridge data repository (https://doi.org/10.17863/CAM. 23316). 


\section{ACKNOWLEDGMENTS}

This work was financially supported by a grant from the European Research Council through an ERC Starting Grant (grant no. ERC-2014-STG-639526, NANOGEN). S.K.-N. acknowledges the support from the EPSRC grant "Centre for Advanced Materials for Integrated Energy Systems (CAMIES)” EP/P007767/1. C.O. thanks the Cambridge Trust and China Scholarship Council for the studentship support. Q.J. is grateful for the financial support through a Marie Sklodowska Curie Fellowship (H2020-MSCA-IF-2015-702868). T.B. thanks the EPSRC Cambridge NanoDTC (EP/L015978/1) for studentship funding. The authors are grateful to Michael Smith for his help with aerosol-jet printing, to Dr Sam Crossley and Chess Boughey for their help with LabVIEW data acquisition and to Dr Thomas Bennett for access to equipment for materials synthesis.

\section{REFERENCES}

(1) Ou, C.; Sanchez-Jimenez, P. E.; Datta, A.; Boughey, F. L.; Whiter, R. A.; Sahonta, S.-L.; Kar-Narayan, S. Template-Assisted Hydrothermal Growth of Aligned Zinc Oxide Nanowires for Piezoelectric Energy Harvesting Applications. ACS Appl. Mater. Interfaces 2016, 8, 13678-13683.

(2) Datta, A.; Choi, Y. S.; Chalmers, E.; Ou, C.; Kar-Narayan, S. Piezoelectric Nylon-11 Nanowire Arrays Grown by Template Wetting for Vibrational Energy Harvesting Applications. Adv. Funct. Mater. 2017, 27, 1604262.

(3) Vining, C. B. An Inconvenient Truth about Thermoelectrics. Nat. Mater. 2009, 8, 83-85.

(4) Chen, Y.; Zhao, Y.; Liang, Z. Solution Processed Organic Thermoelectrics: Towards Flexible Thermoelectric Modules. Energy Environ. Sci. 2015, 8, 401-422.

(5) Zhang, Q.; Sun, Y.; Xu, W.; Zhu, D. Organic Thermoelectric Materials: Emerging Green Energy Materials Converting Heat to Electricity Directly and Efficiently. Adv. Mater. 2014, 26, 6829-6851.

(6) McGrail, B. T.; Sehirlioglu, A.; Pentzer, E. Polymer Composites for Thermoelectric Applications. Angew. Chem., Int. Ed. 2015, 54, $1710-1723$.

(7) Suarez, F.; Nozariasbmarz, A.; Vashaee, D.; Öztürk, M. C. Designing Thermoelectric Generators for Self-Powered Wearable Electronics. Energy Environ. Sci. 2016, 9, 2099-2113.

(8) Du, Y.; Cai, K.; Chen, S.; Wang, H.; Shen, S. Z.; Donelson, R.; Lin, T. Thermoelectric Fabrics: Toward Power Generating Clothing. Sci. Rep. 2015, 5, 6411.

(9) Snyder, G. J.; Toberer, E. S. Complex Thermoelectric Materials. Nat. Mater. 2008, 7, 105-114.

(10) Nozariasbmarz, A.; Zamanipour, Z.; Norouzzadeh, P.; Krasinski, J. S.; Vashaee, D. Enhanced Thermoelectric Performance in a Metal/ Semiconductor Nanocomposite of Iron Silicide/Silicon Germanium. RSC Adv. 2016, 6, 49643-49650.

(11) Koumoto, K.; Funahashi, R.; Guilmeau, E.; Miyazaki, Y.; Weidenkaff, A.; Wang, Y.; Wan, C. Thermoelectric Ceramics for Energy Harvesting. J. Am. Ceram. Soc. 2013, 96, 1-23.

(12) Lin, Y.; Norman, C.; Srivastava, D.; Azough, F.; Wang, L.; Robbins, M.; Simpson, K.; Freer, R.; Kinloch, I. A. Thermoelectric Power Generation from Lanthanum Strontium Titanium Oxide at Room Temperature through the Addition of Graphene. ACS Appl. Mater. Interfaces 2015, 7, 15898-15908.

(13) Russ, B.; Glaudell, A.; Urban, J. J.; Chabinyc, M. L.; Segalman, R. A. Organic Thermoelectric Materials for Energy Harvesting and Temperature Control. Nat. Rev. Mater. 2016, 1, 16050.

(14) Wan, C.; Gu, X.; Dang, F.; Itoh, T.; Wang, Y.; Sasaki, H.; Kondo, M.; Koga, K.; Yabuki, K.; Snyder, G. J.; Yang, R.; Koumoto, K. Flexible N-Type Thermoelectric Materials by Organic Intercalation of Layered Transition Metal Dichalcogenide TiS2. Nat. Mater. 2015, 14, 622-627.
(15) Wan, C.; Tian, R.; Kondou, M.; Yang, R.; Zong, P.; Koumoto, K. Ultrahigh Thermoelectric Power Factor in Flexible Hybrid Inorganic-Organic Superlattice. Nat. Commun. 2017, 8, 1024.

(16) He, M.; Qiu, F.; Lin, Z. Towards High-Performance PolymerBased Thermoelectric Materials. Energy Environ. Sci. 2013, 6, 13521361.

(17) Kim, G.-H.; Shao, L.; Zhang, K.; Pipe, K. P. Engineered Doping of Organic Semiconductors for Enhanced Thermoelectric Efficiency. Nat. Mater. 2013, 12, 719-723.

(18) Bubnova, O.; Khan, Z. U.; Malti, A.; Braun, S.; Fahlman, M.; Berggren, M.; Crispin, X. Optimization of the Thermoelectric Figure of Merit in the Conducting Polymer Poly(3,4-Ethylenedioxythiophene). Nat. Mater. 2011, 10, 429-433.

(19) Taroni, P. J.; Hoces, I.; Stingelin, N.; Heeney, M.; Bilotti, E. Thermoelectric Materials: A Brief Historical Survey from Metal Junctions and Inorganic Semiconductors to Organic Polymers. Isr. J. Chem. 2014, 54, 534-552.

(20) Yu, C.; Kim, Y. S.; Kim, D.; Grunlan, J. C. Thermoelectric Behavior of Segregated-Network Polymer Nanocomposites. Nano Lett. 2008, 8, 4428-4432.

(21) Snyder, G. J.; Snyder, A. H. Figure of Merit ZT of a Thermoelectric Device Defined from Materials Properties. Energy Environ. Sci. 2017, 10, 2280-2283.

(22) Bae, E. J.; Kang, Y. H.; Jang, K.-S.; Cho, S. Y. Enhancement of Thermoelectric Properties of PEDOT: PSS and Tellurium-PEDOT: PSS Hybrid Composites by Simple Chemical Treatment. Sci. Rep. 2016, 6, 18805.

(23) Zhang, B.; Sun, J.; Katz, H. E.; Fang, F.; Opila, R. L. Promising Thermoelectric Properties of Commercial PEDOT:PSS Materials and Their Bi 2 Te 3 Powder Composites. ACS Appl. Mater. Interfaces 2010, 2, 3170-3178.

(24) Søndergaard, R. R.; Hösel, M.; Espinosa, N.; Jørgensen, M.; Krebs, F. C. Practical Evaluation of Organic Polymer Thermoelectrics by Large-Area R2R Processing on Flexible Substrates. Energy Sci. Eng. 2013, 1, 81-88.

(25) Li, J.; Du, Y.; Jia, R.; Xu, J.; Shen, S. Thermoelectric Properties of Flexible PEDOT:PSS/Polypyrrole/Paper Nanocomposite Films. Materials 2017, 10, 780.

(26) Datta, A.; Sangle, A.; Hardingham, N.; Cooper, C.; Kraan, M.; Ritchie, D.; Narayan, V.; Kar-Narayan, S. Structure and Thermoelectric Properties of Bi2-xSbxTe3 Nanowires Grown in Flexible Nanoporous Polycarbonate Templates. Materials 2017, 10, 553.

(27) Dresselhaus, M. S.; Chen, G.; Tang, M. Y.; Yang, R. G.; Lee, H.; Wang, D. Z.; Ren, Z. F.; Fleurial, J.-P.; Gogna, P. New Directions for Low-Dimensional Thermoelectric Materials. Adv. Mater. 2007, 19, 1043-1053.

(28) Datta, A.; Paul, J.; Kar, A.; Patra, A.; Sun, Z.; Chen, L.; Martin, J.; Nolas, G. S. Facile Chemical Synthesis of Nanocrystalline Thermoelectric Alloys Based on $\mathrm{Bi}-\mathrm{Sb}-\mathrm{Te}-\mathrm{Se}$. Cryst. Growth Des. 2010, 10, 3983-3989.

(29) Poudel, B.; Hao, Q.; Ma, Y.; Lan, Y.; Minnich, A.; Yu, B.; Yan, X.; Wang, D.; Muto, A.; Vashaee, D.; Chen, X.; Liu, J.; Dresselhaus, M. S.; Chen, G.; Ren, Z. High-Thermoelectric Performance of Nanostructured Bismuth Antimony Telluride Bulk Alloys. Science 2008, 320, 634-638.

(30) Chen, Y.; He, M.; Liu, B.; Bazan, G. C.; Zhou, J.; Liang, Z. Bendable N-Type Metallic Nanocomposites with Large Thermoelectric Power Factor. Adv. Mater. 2017, 29, 1604752.

(31) Bubnova, O.; Khan, Z. U.; Wang, H.; Braun, S.; Evans, D. R.; Fabretto, M.; Hojati-Talemi, P.; Dagnelund, D.; Arlin, J.-B.; Geerts, Y. H.; Desbief, S.; Breiby, D. W.; Andreasen, J. W.; Lazzaroni, R.; Chen, W. M.; Zozoulenko, I.; Fahlman, M.; Murphy, P. J.; Berggren, M.; Crispin, X. Semi-Metallic Polymers. Nat. Mater. 2014, 13, 190-194.

(32) Park, S. H.; Jo, S.; Kwon, B.; Kim, F.; Ban, H. W.; Lee, J. E.; Gu, D. H.; Lee, S. H.; Hwang, Y.; Kim, J.-S.; Hyun, D.-B.; Lee, S.; Choi, K. J.; Jo, W.; Son, J. S. High-Performance Shape-Engineerable Thermoelectric Painting. Nat. Commun. 2016, 7, 13403. 
(33) Rao, A. M.; Ji, X.; Tritt, T. M. Properties of Nanostructured One-Dimensional and Composite Thermoelectric Materials. MRS Bull. 2006, 31, 218-223.

(34) See, K. C.; Feser, J. P.; Chen, C. E.; Majumdar, A.; Urban, J. J.; Segalman, R. A. Water-Processable Polymer-Nanocrystal Hybrids for Thermoelectrics. Nano Lett. 2010, 10, 4664-4667.

(35) Shi, H.; Liu, C.; Xu, J.; Song, H.; Lu, B.; Jiang, F.; Zhou, W.; Zhang, G.; Jiang, Q. Facile Fabrication of PEDOT: PSS/ Polythiophenes Bilayered Nanofilms on Pure Organic Electrodes and Their Thermoelectric Performance. ACS Appl. Mater. Interfaces 2013, 5, 12811-12819.

(36) Yang, J.; Yip, H.-L.; Jen, A. K.-Y. Rational Design of Advanced Thermoelectric Materials. Adv. Energy Mater. 2013, 3, 549-565.

(37) Lu, Z.; Layani, M.; Zhao, X.; Tan, L. P.; Sun, T.; Fan, S.; Yan, Q.; Magdassi, S.; Hng, H. H. Fabrication of Flexible Thermoelectric Thin Film Devices by Inkjet Printing. Small 2014, 10, 3551-3554.

(38) Jiao, F.; Di, C.-a.; Sun, Y.; Sheng, P.; Xu, W.; Zhu, D. InkjetPrinted Flexible Organic Thin-Film Thermoelectric Devices Based on p- and n-Type Poly(Metal 1,1,2,2-Ethenetetrathiolate)s/Polymer Composites through Ball-Milling. Philos. Trans. R. Soc., A 2014, 372, 20130008.

(39) Wei, Q.; Mukaida, M.; Kirihara, K.; Naitoh, Y.; Ishida, T. Polymer Thermoelectric Modules Screen-Printed on Paper. RSC Adv. 2014, 4, 28802-28806.

(40) Kim, S. J.; We, J. H.; Cho, B. J. A Wearable Thermoelectric Generator Fabricated on a Glass Fabric. Energy Environ. Sci. 2014, 7, $1959-1965$

(41) Cao, Z.; Koukharenko, E.; Tudor, M. J.; Torah, R. N.; Beeby, S. P. Screen Printed Flexible Bi 2 Te 3 -Sb 2 Te 3 Based Thermoelectric Generator. J. Phys.: Conf. Ser. 2013, 476, 012031.

(42) Cao, Z.; Shi, J. J.; Torah, R. N.; Tudor, M. J.; Beeby, S. P. All Dispenser Printed Flexible 3D Structured Thermoelectric Generators. J. Phys.: Conf. Ser. 2015, 660, 012096.

(43) Chen, A.; Madan, D.; Wright, P. K.; Evans, J. W. DispenserPrinted Planar Thick-Film Thermoelectric Energy Generators. J. Micromech. Microeng. 2011, 21, 104006.

(44) He, M.; Zhao, Y.; Wang, B.; Xi, Q.; Zhou, J.; Liang, Z. 3D Printing Fabrication of Amorphous Thermoelectric Materials with Ultralow Thermal Conductivity. Small 2015, 11, 5889-5894.

(45) Orrill, M.; LeBlanc, S. Printed Thermoelectric Materials and Devices: Fabrication Techniques, Advantages, and Challenges. J. Appl. Polym. Sci. 2017, 134, 44256.

(46) Gao, J.; Miao, L.; Liu, C.; Wang, X.; Peng, Y.; Wei, X.; Zhou, J.; Chen, Y.; Hashimoto, R.; Asaka, T.; Koumoto, K. A Novel GlassFiber-Aided Cold-Press Method for Fabrication of n-Type Ag $2 \mathrm{Te}$ Nanowires Thermoelectric Film on Flexible Copy-Paper Substrate. J. Mater. Chem. A 2017, 5, 24740-24748.

(47) Glatz, W.; Schwyter, E.; Durrer, L.; Hierold, C. Bi2Te3-Based Flexible Micro Thermoelectric Generator With Optimized Design. J. Microelectromech. Syst. 2009, 18, 763-772.

(48) Smith, M.; Choi, Y. S.; Boughey, C.; Kar-Narayan, S. Controlling and Assessing the Quality of Aerosol Jet Printed Features for Large Area and Flexible Electronics. Flexible Printed Electron. 2017, 2, 015004

(49) Datta, A.; Nolas, G. S. Composition Controlled Synthesis of Bi Rich Bil-xSbx Alloy Nanocrystals by a Low Temperature Polyol Process. CrystEngComm 2011, 13, 2753-2757.

(50) Scherrer, P. Nachrichten von Der Gesellschaft Der Wissenschaften Zu Göttingen. Göttinger Nachrichten Gesell. 1918, 2, 98-99.

(51) Fan, Z.; Du, D.; Yao, H.; Ouyang, J. Higher PEDOT Molecular Weight Giving Rise to Higher Thermoelectric Property of PEDOT: PSS: A Comparative Study of Clevios P and Clevios PH1000. ACS Appl. Mater. Interfaces 2017, 9, 11732-11738.

(52) Meng, W.; Ge, R.; Li, Z.; Tong, J.; Liu, T.; Zhao, Q.; Xiong, S.; Jiang, F.; Mao, L.; Zhou, Y. Conductivity Enhancement of PEDOT: PSS Films via Phosphoric Acid Treatment for Flexible All-Plastic Solar Cells. ACS Appl. Mater. Interfaces 2015, 7, 14089-14094.
(53) Wei, Q.; Mukaida, M.; Kirihara, K.; Naitoh, Y.; Ishida, T. Recent Progress on PEDOT-Based Thermoelectric Materials. Materials 2015, $8,732-750$.

(54) Zebarjadi, M.; Esfarjani, K.; Dresselhaus, M. S.; Ren, Z. F.; Chen, G. Perspectives on Thermoelectrics: From Fundamentals to Device Applications. Energy Environ. Sci. 2012, 5, 5147-5162.

(55) Bahk, J.-H.; Fang, H.; Yazawa, K.; Shakouri, A. Flexible Thermoelectric Materials and Device Optimization for Wearable Energy Harvesting. J. Mater. Chem. C 2015, 3, 10362-10374.

(56) Du, Y.; Cai, K. F.; Chen, S.; Cizek, P.; Lin, T. Facile Preparation and Thermoelectric Properties of Bi2Te3 Based Alloy Nanosheet/ PEDOT: PSS Composite Films. ACS Appl. Mater. Interfaces 2014, 6, 5735-5743.

(57) Yee, S. K.; Coates, N. E.; Majumdar, A.; Urban, J. J.; Segalman, R. A. Thermoelectric Power Factor Optimization in PEDOT:PSS Tellurium Nanowire Hybrid Composites. Phys. Chem. Chem. Phys. 2013, 15, 4024-4032.

(58) Zhao, L.-D.; Lo, S.-H.; Zhang, Y.; Sun, H.; Tan, G.; Uher, C.; Wolverton, C.; Dravid, V. P.; Kanatzidis, M. G. Ultralow Thermal Conductivity and High Thermoelectric Figure of Merit in SnSe Crystals. Nature 2014, 508, 373-377.

(59) Venkatasubramanian, R.; Siivola, E.; Colpitts, T.; O’Quinn, B. Thin-Film Thermoelectric Devices with High Room-Temperature Figures of Merit. Nature 2001, 413, 597-602.

(60) Minnich, A. J.; Dresselhaus, M. S.; Ren, Z. F.; Chen, G. Bulk Nanostructured Thermoelectric Materials: Current Research and Future Prospects. Energy Environ. Sci. 2009, 2, 466-479.

(61) Wei, Q.; Mukaida, M.; Kirihara, K.; Naitoh, Y.; Ishida, T. Polymer Thermoelectric Modules Screen-Printed on Paper. RSC Adv. 2014, 4, 28802-28806.

(62) Culebras, M.; Gómez, C.; Cantarero, A. Review on Polymers for Thermoelectric Applications. Materials 2014, 7, 6701-6732. 